\title{
Reduction of conspicuous facial pores by topical fullerene: possible role in the suppression of $\mathrm{PGE}_{2}$ production in the skin
}

\author{
Shigeki Inui ${ }^{i^{*}}$, Ayako Mori ${ }^{2}$, Masayuki Ito ${ }^{3}$, Sayuri Hyodo ${ }^{3}$ and Satoshi Itami ${ }^{1}$
}

\begin{abstract}
Background: Conspicuous facial pores are therapeutic targets for cosmeceuticals. Here we examine the effect of topical fullerene on conspicuous facial pores using a new image analyser called the VISIA ${ }^{\oplus}$ system. Ten healthy Japanese females participated in this study, and they received applications of $1 \%$ fullerene lotion to the face twice a day for 8 weeks.
\end{abstract}

Findings: Fullerene lotion significantly decreased conspicuous pores by $17.6 \%$ ( $p<0.05$, Wilcoxon signed-rank test) after an 8-week treatment. A self-administered questionnaire indicated that this reduction achieved cosmetically appreciable effects. In addition, to investigate the mechanism of effect of fullerene, we examined its effect on UVB-induced prostaglandin $\mathrm{E}_{2}\left(\mathrm{PGE}_{2}\right)$ production in reconstructed human epidermis (RhE). The results showed that irradiation of RhE with $1000 \mathrm{~mJ} / \mathrm{cm}^{2}$ increased $P G E_{2}$ production by $62.3 \%(p<0.05$, Mann-Whitney $U$-test) and the addition of $28 \mu \mathrm{M}$ fullerene significantly suppressed the UVB-induced $\mathrm{PGE}_{2}$ production by $18.3 \%(p<0.05)$.

Conclusions: Fullerene lotion significantly decreases conspicuous facial pores after an 8-week treatment possibly through the suppression of $\mathrm{PGE}_{2}$ production in the epidermis.

Keywords: Fullerene, Facial pore, Prostaglandin $E_{2}$, Cosmeceutical, Melanin

\section{Background}

Conspicuous facial pores are therapeutic targets for cosmeceuticals. Previously it has been reported that the topical vitamin C derivative ascorbyl 2-phosphate 6-palmitate (APPS) decreased conspicuous facial pores [1]. Here we examine the effect of topical fullerene on conspicuous facial pores using the new image analszer called the $\mathrm{VISIA}^{\circ}$ system (Canfield Scientific Inc., Fairfield, NJ).

\section{Methods}

Ten healthy Japanese females participated in this study between January and June of 2013 at Clinic Mori (Minato-ku, Tokyo). Age range of participants was from 39 to 70 years and the mean age was 46 years. After an informed consent was obtained, $1 \%$ fullerene lotion (Radical Sponge $1.0 \%, 2$ phenoxyethanol $0.5 \%$, glycerine $5.0 \%$, 1,3-butylene glycol $5.0 \%$, essential oil as needed, water q.s. to $100 \%$ ) was

\footnotetext{
* Correspondence: inui@r-derma.med.osaka-u.ac.jp

'Department of Regenerative Dermatology, Graduate School of Medicine,

Osaka University, 2-2, G2, Yamadaoka, Suita -shi, Osaka 565-0871, Japan

Full list of author information is available at the end of the article
}

applied on the face twice a day for 8 weeks. The subjects were not permitted to use sunscreen products. However, they were allowed to use ordinary cosmetics but not medicinal cosmetics. The high-resolution analysing system VISIA-CR (Canfield, Fairfield, NJ) was used for computer analysis to precisely measure conspicuous pores through analysis scripts [2]. The photographs from VISIA-CR were analysed by a pores analysis algorithm in Vaestro image analysis toolkit, v 2.0 (Canfield, Fairfield, NJ) and subsequently the visible conspicuous facial pores were quantitatively measured. The analysis was carried out separately on the right and left sides of the face and the results were combined to get the total pore count. In principle, the pores were recognised as circular areas darker than the surrounding skin tone and much smaller than skin spots. This study was approved by the institutional ethics committee. An informed consent was obtained from all patients before enrolment in the study. To examine the effect of fullerene on UVB-induced prostaglandin $\mathrm{E}_{2}\left(\mathrm{PGE}_{2}\right)$ production in reconstructed human epidermis (RhE) LabCyte EPIMODEL24 (Japan Tissue Engineering Co. Ltd, Gamagori, 
Japan), RhE was incubated in $500 \mu \mathrm{l}$ of the assay medium of the kit on the 24-well plate for 1 hour at $37^{\circ} \mathrm{C}$ in a humidified atmosphere containing $5 \% \mathrm{CO}_{2}$. Then, 2.8 or 28 $\mu \mathrm{M}$ water-soluble polyvinylpyrrolidone (PVP)-wrapped fullerene or a mock solution (1\% PVP in PBS) was added. After incubation for 1 hour, the cultures were irradiated with $1000 \mathrm{~mJ} / \mathrm{cm}^{2}$ UVB. Thirty minutes later, the medium was aspirated and the cells were washed three times with PBS. The cultures were kept in the fresh medium for 6 hours and the conditioned medium was finally subjected to $\mathrm{PGE}_{2}$ ELISA ( $\mathrm{PGE}_{2}$ EIA Monoclonal Kit, Cayman Chemical Company, Michigan). A statistical analysis was performed using Wilcoxon signed-rank test or Mann-Whitney $U$-test. A difference with a $\mathrm{p}$ value of $<0.05$ was considered significant.

\section{Results and discussion}

The mean number of conspicuous facial pores before and after the fullerene lotion treatment is shown in Figure 1a. The fullerene lotion significantly decreased the conspicuous pores by $17.6 \%(\mathrm{p}<0.05$, Wilcoxon signed-rank test) after an 8-week treatment. Because this reduction seemed moderately significant to establish cosmetic efficiency, we administered a self-administered questionnaire, which inquired regarding whether participants felt the effect of the tested lotion on opening and pigmentation of pores, facial skin texture and the general condition of facial skin. Among the 10 subjects enrolled, 7 felt an improvement of texture and general condition of facial skin, and 6 felt that there was improvement of the opening and pigmentation of pores; indicating that the fullerene lotion achieved appreciable cosmetic effect. The representative case is shown in Figure 1b, which demonstrates a reduction in the conspicuous pores on the cheek. Using dermoscopy, the regression of the conspicuous pores (triangles) was observed in the same case (Figure 1c). It has been previously reported that skin pigmentation can be classified into three main patterns, i.e., spotty, globular and elongated types, using a video camera equipped with an UV-emitting unit [3]. Moreover, the spotty type corresponds to a perifollicular pattern where the dark spots are round and evenly distributed, and it is decreased by depigmenting formulations such as azelaic acid and the soy extract, resulting in skin lighting [3]. In addition, we examined the effect of topical APPS on the conspicuous facial pores using another highresolution digital camera system called the Robo-skin analyser RSA-50 (Inforward Co, Tokyo, Japan) [1]. It was seen that APPS treatment significantly decreased the conspicuous and blackish pores but not the open pores after a 4-week treatment, indicating that APPS can decrease conspicuous pores mainly by whitening them. Collectively, the regression of conspicuous pores can be ascribed to the suppression of perifollicular melanogenesis. Accordingly, to investigate the mechanism of effect of fullerene, its effect was examined on UVB-induced $\mathrm{PGE}_{2}$ production in RhE because $\mathrm{PGE}_{2}$ reportedly stimulates tyrosinase and dendricity of melanocytes $[4,5]$. Irradiation with $1000 \mathrm{~mJ} / \mathrm{cm}^{2}$ increased $\mathrm{PGE}_{2}$ production in RhE by $62.3 \%$ ( $<<0.05$, Mann-Whitney $U$-test). However, the addition of $28 \mu \mathrm{M}$ fullerene significantly suppressed the UVB-induced $\mathrm{PGE}_{2}$ production by $18.3 \%(\mathrm{p}<0.05)$ (Figure 2). Therefore, we suggest that the suppression of $\mathrm{PGE}_{2}$ in the epidermis

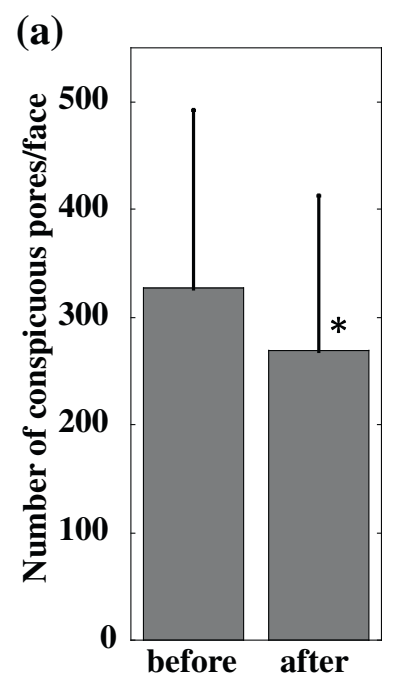

(b)

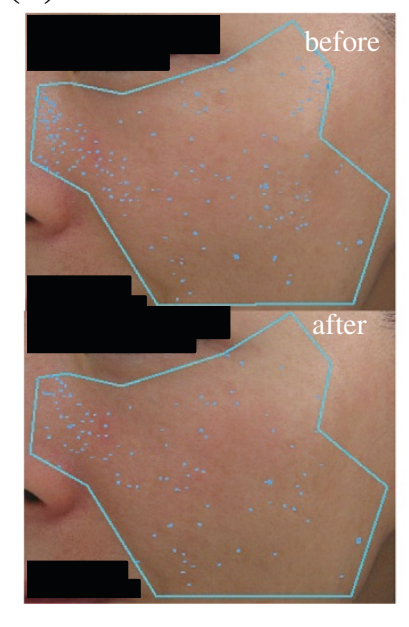

(c)

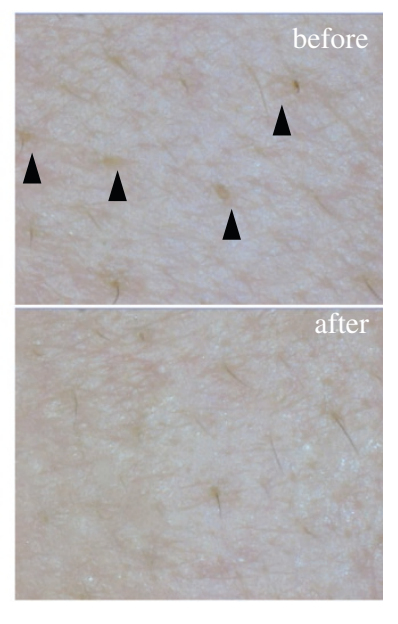

Figure 1 Effect of fullerene on conspicuous facial pores. (a) Reduction of the conspicuous facial pores after fullerene treatment for 8 weeks. The graph shows the mean \pm standard deviation $(n=10)$. ${ }^{*} p<0.05$, Wilcoxon signed-rank test. (b) The photos of conspicuous pores on the cheek by VISIA ${ }^{\oplus}$ in the representative case (43-year-old female) before (upper panel) and after fullerene treatment for 8 weeks (lower panel) are shown. The number of conspicuous pores has decreased from 170 to 103. (c) Using dermoscopy, a regression of conspicuous pores (triangles) is observed in the same case. 


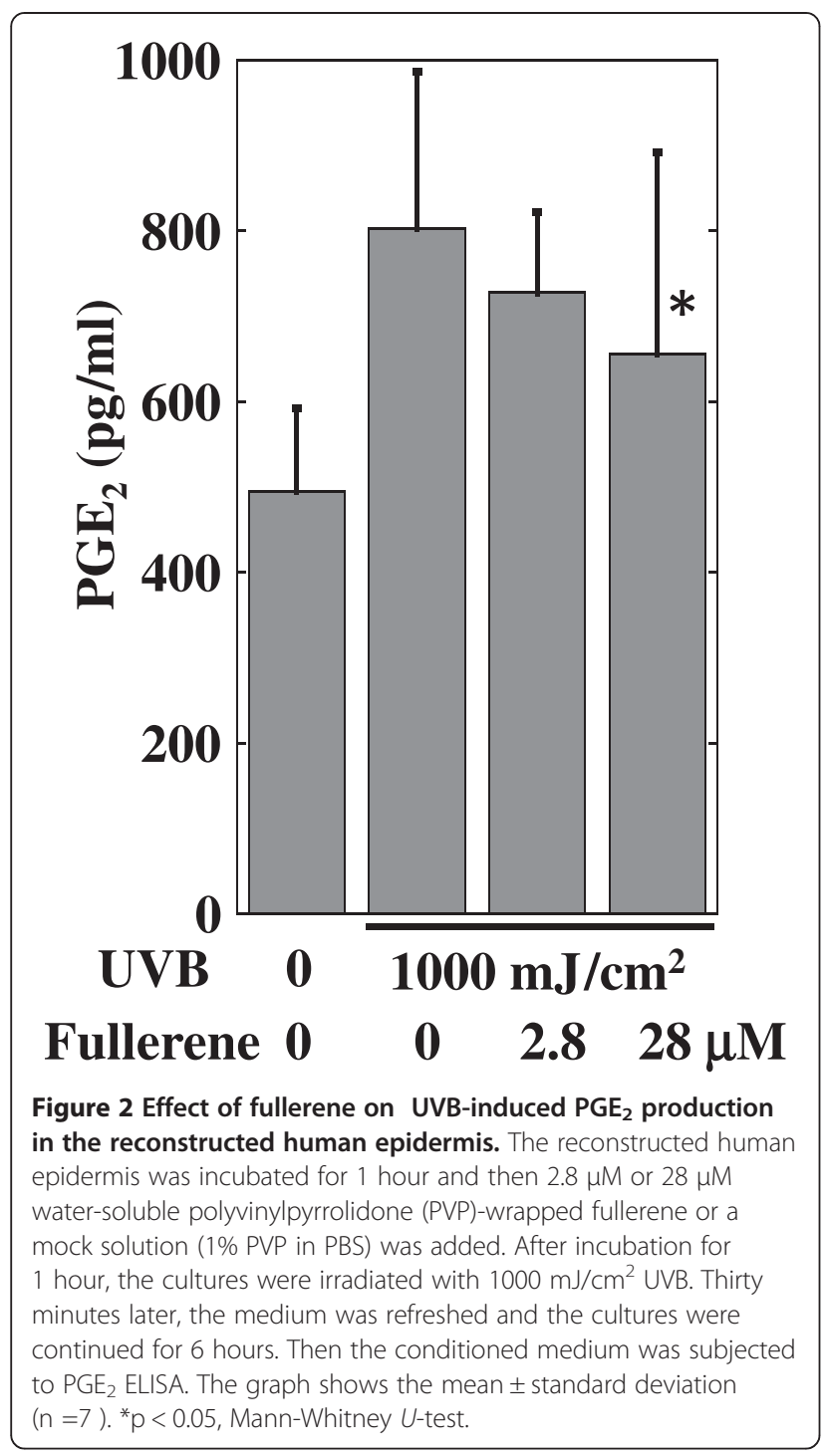

is one of the potential pathways for the effect of fullerene on melanogenesis, which is associated with the pathogenesis of conspicuous pores. In addition, water-soluble PVP-wrapped fullerene reportedly shows an appreciable absorbance in the UVB spectrum (290-320nm) [6], suggesting that the effect of fullerene on $\mathrm{PGE}_{2}$ may be partially through UV absorption.

Here, we utilised the LabCyte EPI-MODEL24 but this model does not contain melanocytes. Therefore, our assays reflected only fullerene effect on epidermal keratinocytes. On the other hand, fullerene has been previously reported to inhibit UVA-induced melanogenesis from cultured human melanocytes [6]. Collectively, keratinocytes and melanocytes both are suggested targets of fullerene. However, to explore the effect of fullerene on $\mathrm{PGE}_{2}$ production more precisely, it is of interest to study of animal models or even ex-vivo human skin in the near future.
Because even a dose of $80 \mathrm{~mJ} / \mathrm{cm}^{2}$ UVB is reportedly sufficient for induction of $\mathrm{PGE}_{2}$ production in cultured human keratinocytes [7], the dose of UVB used for irradiation in this study $\left(1000 \mathrm{~mJ} / \mathrm{cm}^{2}\right)$ was extremely high. Likewise, the mean of minimal erythema dose of UVB for Japanese is $43.7 \mathrm{~mJ} / \mathrm{cm}^{2}$ [8]; therefore, the UVB dose utilised here is remarkably intense. However, previously it was reported that, whereas only $5 \mathrm{~mJ} / \mathrm{cm}^{2} \mathrm{UVB}$ could stimulate co-culture of human keratinocytes and melanocytes, a $100 \mathrm{~mJ} / \mathrm{cm}^{2} \mathrm{UVB}$ dose was required to induce melanogenesis in human pigmented reconstructed epidermis. This indicates that the reconstructed epidermis is more resistant to the UVB effect than cultured epidermal cells [9], supporting our observation of resistance or insensitivity of the RhE to UVB.

In conclusion, the fullerene lotion significantly decreased the conspicuous facial pores after an 8-week treatment. Further, the experimental data suggests that the suppression of $\mathrm{PGE}_{2}$ production in epidermis is one of potential pathways for this cosmetic effect of fullerene.

\section{Abbreviations}

APPS: Ascorbyl 2-phosphate 6-palmitate; $P_{\text {GE }}$ : Prostaglandin $E_{2 i}$ RhE: Reconstructed human epidermis; PVP: Polyvinylpyrrolidone.

\section{Competing interests}

The cost of this study was covered by Vitamin C60 BioResearch Corporation. The authors $\mathrm{Ml}$ and $\mathrm{SH}$ belong to this company.

\section{Authors' contributions}

S Inui and S Itami designed and organized the whole study. S Inui analyzed the data and wrote the paper. AM carried out the clinical study. MI and SH performed the in vitro experiments. All authors read and approved the final manuscript.

\section{Author details}

'Department of Regenerative Dermatology, Graduate School of Medicine, Osaka University, 2-2, G2, Yamadaoka, Suita -shi, Osaka 565-0871, Japan.

${ }^{2}$ Clinic Mori, 1-1-5, Motoakasaka, Minato-ku, Tokyo 107-0051, Japan. ${ }^{3}$ Vitamin C60 BioResearch Corporation, 1-3-19, Yaesu, Chuo-ku, Tokyo 103-0028, Japan.

Received: 27 December 2013 Accepted: 15 February 2014 Published: 22 February 2014

\section{References}

1. Inui S, Itami S: Perifollicular pigmentation is the first target for topical vitamin C derivative ascorbyl 2-phosphate 6-palmitate (APPS): randomized, single-blinded, placebo-controlled study. J Dermatol 2007, 34:221-223.

2. Saedi N, Petrell K, Arndt K, Dover J: Evaluating facial pores and skin texture after low-energy nonablative fractional 1440-nm laser treatments. J Am Acad Dermatol 2013, 68:113-118.

3. Hermanns JF, Petit L, Martalo O, Pierard-Franchimont C, Cauwenbergh G, Pierard GE: Unraveling the patterns of subclinical pheomelanin-enriched facial hyperpigmentation: effect of depigmenting agents. Dermatology 2000, 201:118-122.

4. Scott G, Leopardi S, Printup S, Malhi N, Seiberg M, Lapoint R: Proteinase-activated receptor-2 stimulates prostaglandin production in keratinocytes: analysis of prostaglandin receptors on human melanocytes and effects of PGE2 and PGF2alpha on melanocyte dendricity. J Invest Dermatol 2004, 122:1214-1224.

5. Starner RJ, McClelland L, Abdel-Malek Z, Fricke A, Scott G: PGE(2) is a UVR-inducible autocrine factor for human melanocytes that stimulates tyrosinase activation. Exp Dermatol 2010, 19:682-684. 
6. Xiao L, Matsubayashi K, Miwa N: Inhibitory effect of the water-soluble polymer-wrapped derivative of fullerene on UVA-induced melanogenesis via downregulation of tyrosinase expression in human melanocytes and skin tissues. Arch Dermatol Res 2007, 299:245-257.

7. Terazawa S, Nakajima H, Shingo M, Niwano T, Imokawa G: Astaxanthin attenuates the UVB-induced secretion of prostaglandin E2 and interleukin-8 in human keratinocytes by interrupting MSK1 phosphorylation in a ROS depletion-independent manner. Exp Dermatol 2012,

21(Suppl 1):11-17.

8. Kawada A, Noda T, Hiruma M, Ishibashi A, Arai S: The relationship of sun protection factor to minimal erythema dose, Japanese skin type, and skin color. J Dermatol 1993, 20:514-516.

9. Duval C, Regnier M, Schmidt R: Distinct melanogenic response of human melanocytes in mono-culture, in co-culture with keratinocytes and in reconstructed epidermis, to UV exposure. Pigment Cell Res 2001, 14:348-355

doi:10.1186/1477-3155-12-6

Cite this article as: Inui et al:: Reduction of conspicuous facial pores by topical fullerene: possible role in the suppression of $\mathrm{PGE}_{2}$ production in the skin. Journal of Nanobiotechnology 2014 12:6.

\section{Submit your next manuscript to BioMed Central and take full advantage of:}

- Convenient online submission

- Thorough peer review

- No space constraints or color figure charges

- Immediate publication on acceptance

- Inclusion in PubMed, CAS, Scopus and Google Scholar

- Research which is freely available for redistribution

Submit your manuscript at www.biomedcentral.com/submit

C Biomed Central 